The Astrophysical Journal, 202: 407-414, 1975 December 1

(C) 1975. The American Astronomical Society. All rights reserved. Printed in U.S.A.

\title{
OBSERVATIONS OF AN ISOLATED COMPACT INFRARED SOURCE IN PERSEUS
}

\author{
W. E. Westbrook, * E. E. Becklin, $\dagger \ddagger$ K. M. Merrill, $\S$ G. Neugebauer, $\dagger$ M. Schmidt, $\dagger$ \\ S. P. Willner, * AND C. G. WyNN-WILliams $\$ \|$ \\ Received 1975 March 27; revised 1975 May 27
}

\begin{abstract}
Infrared, optical, and radio observations are described of an unusual compact infrared source discovered during the AFCRL survey. It is suggested that the object may be a planetary nebula seen at an unprecedentedly early phase in its evolution.
\end{abstract}

Subject headings: infrared sources - planetary nebulae

\section{INTRODUCTION}

Bill Westbrook, a graduate student in the Caltech infrared astronomy group, was engaged during 1972 on a study of previously unidentified sources from the AFCRL catalog. On 1975 June 4, while this paper was in press, he died at the age of 26 after a serious illness. The paper is a report of ground-based infrared, optical and radio studies of the most interesting of his sources, CRL 618.

\section{OBSERVATIONS}

\section{a) Identification and Diameter of Infrared Source}

The Air Force Cambridge Research Laboratory rocket-borne infrared survey at 4,11 , and $20 \mu$ (Price and Walker, private communication) contains a number of previously unidentified sources. The source CRL 618 is a bright infrared object which appears to be associated with a pair of small visual nebulosities each of which has a diameter of $2^{\prime \prime}-3$ ". The eastern visual component appears circular and 3 or 4 times brighter than the western component which is irregular. The fainter visual object is $7^{\prime \prime} \pm 1^{\prime \prime}$ west and within $1^{\prime \prime}$ in declination of the brighter component, and the infrared source lies between the two visual features. On the red Palomar Sky Survey plate (see Fig. 1) both objects appear stellar and isolated from other regions of nebulosity.

The absolute position of the infrared source, measured with respect to nearby field stars, is (1950.0)

$$
\alpha=4^{\mathrm{h}} 39^{\mathrm{m}} 33 \mathrm{~s} 8 \pm 0 \$ 1, \quad \delta=36^{\circ} 1^{\prime} 15^{\prime \prime} \pm 1^{\prime \prime} .
$$

In addition, the position of the infrared source was determined at the 200 -inch ( $5 \mathrm{~m}$ ) Hale telescope with a

* George W. Downs Laboratory of Physics, California Institute of Technology.

$\dagger$ Hale Observatories, California Institute of Technology, Carnegie Institution of Washington.

$\ddagger$ Guest observer, Mauna Kea Observatory, Institute for Astronomy, University of Hawaii.

$\S$ Department of Physics, University of California, San Diego.

|| Mullard Radio Astronomy Observatory, Cavendish Laboratory, Cambridge, England. $2^{\prime \prime}$ aperture to be $3^{\prime \prime} \pm 1^{\prime \prime}$ west and within $1^{\prime \prime}$ in declination of the bright eastern visual nebulosity. The galactic coordinates of the object are $l^{\mathrm{II}}=166^{\circ} .5$, $b^{\text {II }}=-6.5$.

Spatial scans of the infrared source were made in a north-south direction on the 200-inch telescope at $11.2 \mu(\Delta \lambda=1.5 \mu)$ with a $0.5 \times 4^{\prime \prime}$ slit aperture. These scans show that the source is extended with the best fit to a Gaussian source profile having a full width at half-maximum diameter of $0.4 \pm 0$ ". 2 . Observations with a $24^{\prime \prime}$ aperture and $64^{\prime \prime}$ chopping spacing showed no evidence of any low-brightness halo at either $10 \mu$ or $20 \mu$, or of more than one infrared source.

\section{b) Infrared Photometry and Spectroscopy}

Infrared observations were carried out on several nights in 1973 and 1974 at the Mount Wilson 60-inch $(1.5 \mathrm{~m})$, the Hale 200-inch, and the Mauna Kea 88-inch $(2.2 \mathrm{~m})$ telescopes using conventional infrared photometers. A summary of broad-band infrared measurements is given in Table 1; Figure 2 shows a plot of the object's energy distribution. With a diameter of 0.4 , the object's $10-\mu$ brightness temperature is $225 \mathrm{~K}$; the color temperature implied by the data of Figure 2 is $275 \mathrm{~K}$, and the emissivity at $10 \mu$ is thus on the order of 0.3 .

Figure 3 shows the 8 to $13 \mu$ spectrum of the source obtained with a $22^{\prime \prime}$ beam during 1973 December using

TABLE 1

INFRARED PHOTOMETRY

\begin{tabular}{rrrc}
\hline$\lambda$ & $\Delta \lambda$ & \multicolumn{1}{c}{ Mag* $^{*}$} & $\log S_{\nu}\left(\mathrm{W} \mathrm{m}^{-2} \mathrm{~Hz}^{-1}\right)$ \\
\hline $1.65 \ldots \ldots$ & 0.3 & $11.6 \pm 0.1$ & -27.67 \\
$2.2 \ldots \ldots$ & 0.4 & $9.3 \pm 0.1$ & -26.95 \\
$3.5 \ldots \ldots$ & 0.6 & $5.3 \pm 0.1$ & -25.69 \\
$4.8 \ldots \ldots$ & 0.6 & $2.7 \pm 0.1$ & -24.92 \\
$8.7 \ldots \ldots$ & 1.2 & $-1.7 \pm 0.2$ & -23.62 \\
$10.1 \ldots \ldots$ & 5.0 & $-2.4 \pm 0.1$ & -23.47 \\
$11.2 \ldots \ldots$ & 1.5 & $-2.5 \pm 0.2$ & -23.50 \\
$12.5 \ldots \ldots$ & 1.3 & $-3.1 \pm 0.2$ & -23.38 \\
$20.0 \ldots \ldots$ & 7.0 & $-4.7 \pm 0.3$ & -23.11 \\
$34.0 \ldots \ldots$ & 10.0 & $-5.6 \pm 0.5$ & -23.22 \\
\hline
\end{tabular}

* Vega has mag $=0.0$. 
TABLE 2

WAVELENGTHS OF EMISSION LINES

\begin{tabular}{|c|c|c|c|}
\hline$\lambda_{\text {obs }}(\AA)$ & Strength & Identification & $\lambda_{0}$ \\
\hline $\begin{array}{l}3506.7 \ldots \ldots \\
3970.6 \ldots \ldots \\
4070.1 \ldots \ldots \\
4100.7 \ldots \ldots \\
4341.8 \ldots \ldots \\
4415.0 \ldots \\
4570.1 \ldots \\
4720.9 \ldots \\
4860.8 \ldots \\
5008.5 \ldots \\
5157.6 \ldots \\
5197.6 \ldots \\
5265.4 \ldots \\
5329.0 \ldots \\
5528.0 \ldots \\
5755.2 \ldots \\
6298.2 \ldots \\
6361.8 \ldots \\
6561.1 \ldots \\
6579.8 \ldots \\
6715.2 \ldots \\
6728.6 \ldots \\
7065.4 \ldots \\
7152.8 \ldots \\
7166.8 \ldots \\
7288.2 \ldots \\
7319.5 \ldots \\
7373.5 \ldots \\
7407.6 \ldots \\
7448.2 \ldots \\
7 \\
\end{array}$ & $\begin{array}{l}1 \\
2 \\
7 \\
2 \\
2 \\
2 \\
4 \\
3 \\
7 \\
2 \\
1 \\
7 \\
1 \\
0 \\
1 \\
2 \\
8 \\
6 \\
6 \\
5 \\
4 \\
6 \\
1 \\
4 \\
2 \\
3 \\
4 \\
3 \\
1 \\
0\end{array}$ & 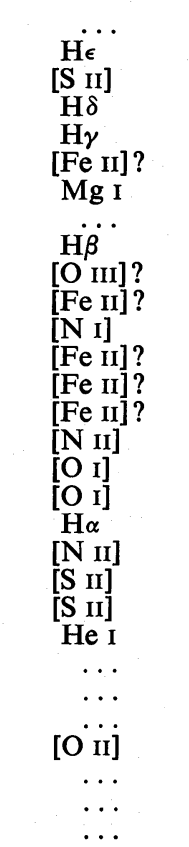 & 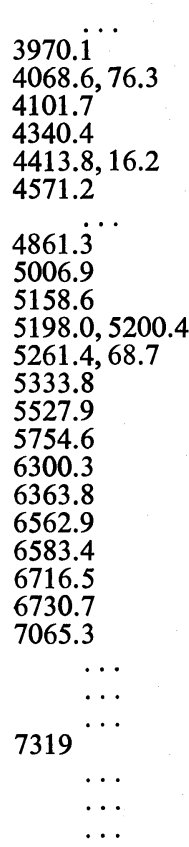 \\
\hline
\end{tabular}

the University of California, San Diego, cooled filterwheel spectrometer at the University of MinnesotaUniversity of California, San Diego, 60-inch infrared telescope on Mount Lemmon. Gillett and Forrest (1973) give a description of the equipment and observing techniques. In this spectral region, the flux $F_{\lambda}$ is nearly constant $\left(F_{v} \propto \nu^{-2}\right)$ at $9.2 \times 10^{-16}$ $\mathrm{W} \mathrm{cm}^{-2} \mu^{-1}$, smoothly rising to about 5 percent above this value near $9.5 \mu$, smoothly falling to 5 percent below this value near $12 \mu$, then rising again. This gradual undulation, which is confirmed by a third independent scan not included here, is more suggestive of temperature variations within the source than of the familiar wavelength-dependent emissivities of silicates or silicon carbide. No spectral lines, such as the 12.78- $\mu$ $\mathrm{Ne}$ II line, are seen above the continuum.

\section{c) Optical Spectroscopy}

Photographic spectra were obtained on several nights in 1973 October and 1974 February with the Cassegrain image-tube spectrograph at the 200-inch telescope. The spectra show numerous emission lines on a continuum and generally are characteristic of an ioniżed gas. The [O I] $\lambda \lambda 6300,6363 ;[\mathrm{N} \mathrm{I}] \lambda \lambda 5198,5200$; and $\mathrm{Mg}$ I $\lambda 4571$ are unusually strong. Table 2 gives the wavelengths of emission lines, with a subjective indication of their strengths; the identification of one line as [O III] $\lambda 5007$ is uncertain. The identifications of [Fe II] are based on the spectrum of $\eta$ Carinae (Thackeray 1953). They are uncertain since equally
TABLE 3

EMisSion-Line FluXes

\begin{tabular}{|c|c|}
\hline Emission Line & $\begin{array}{c}\text { Observed } \\
\left(10^{-15} \mathrm{~W} \mathrm{~m}^{-2}\right)\end{array}$ \\
\hline 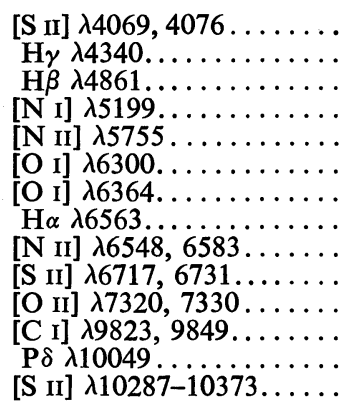 & $\begin{array}{l}0.056 \\
0.017 \\
0.068 \\
0.102 \\
0.014 \\
0.73 \\
0.26 \\
0.63 \\
0.26 \\
0.40 \\
0.08 \\
0.73 \\
0.022 \\
1.04\end{array}$ \\
\hline
\end{tabular}

strong lines of [Fe II] at 4244, 4287, and $5274 \AA$ observed in $\eta$ Carinae are missing. The spectra of the two components are similar with the east component being 3 to 4 times stronger than the west component. The radial velocity of the object relative to the local standard of rest is about $-50 \mathrm{~km} \mathrm{~s}^{-1}$.

Observations made in 1974 January with the 32-channel spectrophotometer on the 200-inch telescope are given in Figure 4. Measurements were made with a 7" aperture and channel widths of $40 \AA$ below $5700 \AA$ and $80 \AA$ above $5700 \AA$. Emission-line fluxes determined from these observations are given in Table 3. In cases where more than one line contributes to a given channel, the photographic spectra were consulted. In addition to lines seen on the photographic spectra, the spectrophotometry shows emission of [C I] $\lambda \lambda 9823,9849 ; \mathrm{P} \delta \lambda 10049$; and [S II] $\lambda \lambda 10287$, $10320,10338,10373$.

\section{d) Optical Polarimetry}

Crude measurements of the visual polarization of the object were obtained in 1974 November using a 7" aperture with an unfiltered S17 photocathode at the 100-inch $(2.5 \mathrm{~m})$ Hooker telescope on Mount Wilson. These measurements were repeated at the 200-inch Hale telescope in 1974 December, when a 10" aperture and a filter and photocathode approximating the $V$ photometric band were used. The measurements agree, and the polarization observed was $21 \pm 2$ percent with the electric vector in position angle $0^{\circ} \pm 6^{\circ}$; i.e., the electric vector is directed along the north-south direction. The position angle was determined only from the geometry of the telescope and instrument. The observations were centered on the bright visible object, but the seeing and sky conditions were such that the fainter object could not be seen as a separate object. The data might therefore contain some contribution from the fainter object.

\section{e) Radio Observations}

Radio continuum observations were made in 1973 December with the Cambridge 5-km radio telescope 


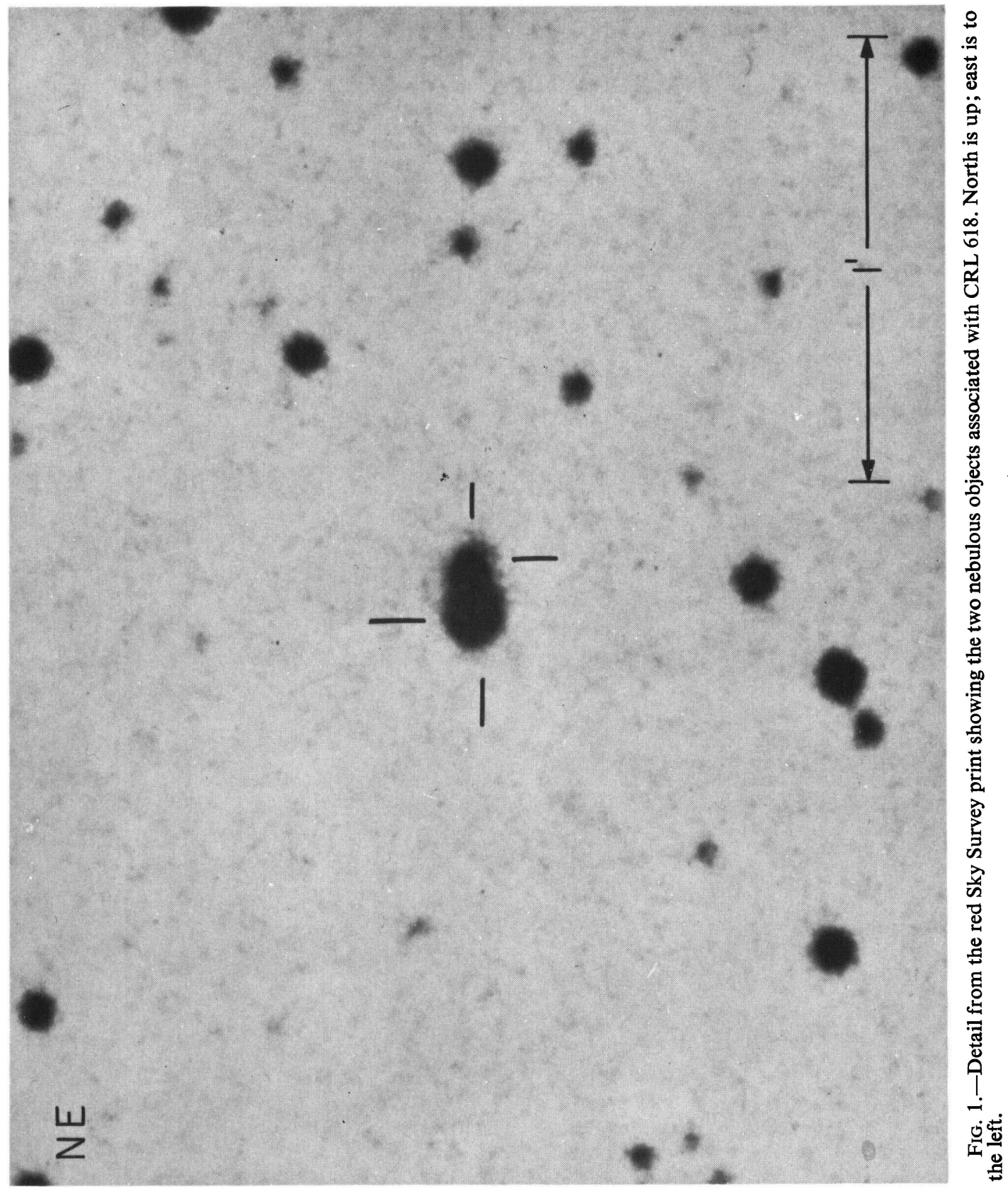




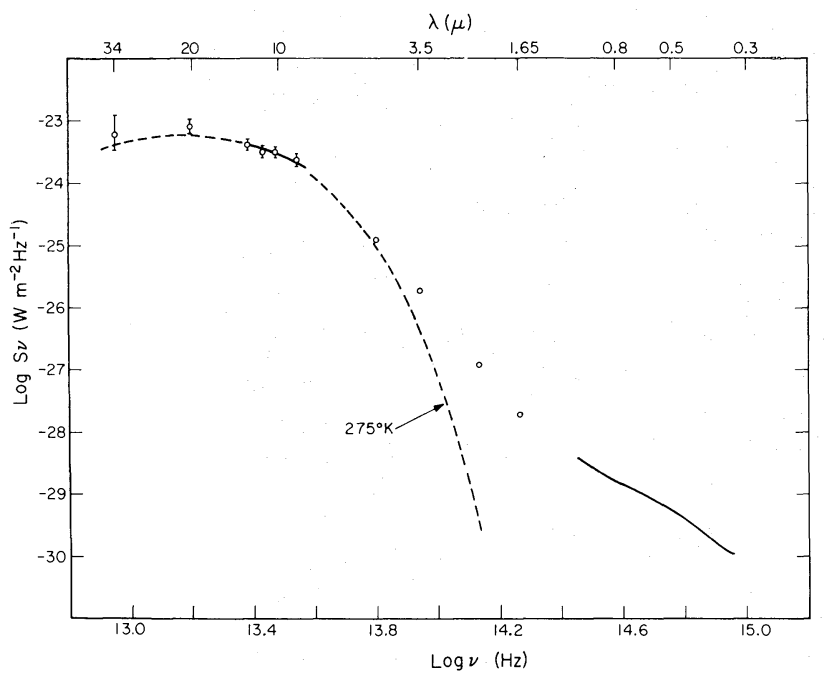

FIG. 2.-Energy distribution showing broad-band infrared measurements (open circles) and infrared and optical spectrophotometric measurements (solid lines). Included also is the energy distribution of a $275 \mathrm{~K}$ blackbody. Infrared points without error bars have errors less than $10 \%$, and the continuum was taken from Figs. 3 and 4.

as described by Ryle (1972). An area $\pm 38^{\prime \prime}$ in declination and $\pm 30^{\prime \prime}$ in right ascension centered on the infrared source was searched at $5 \mathrm{GHz}$ with $2^{\prime \prime}$ eastwest and 3".4 north-south resolution. No emission was detected above a $3 \sigma$ level of $14 \times 10^{-29} \mathrm{~W} \mathrm{~m}^{-2} \mathrm{~Hz}^{-1}$.

\section{DISCUSSION}

\section{a) Geometry of the Object}

The large linear polarization exhibited at visible wavelengths is a strong indication of reflection nebulosity in the source. In support of this idea are the

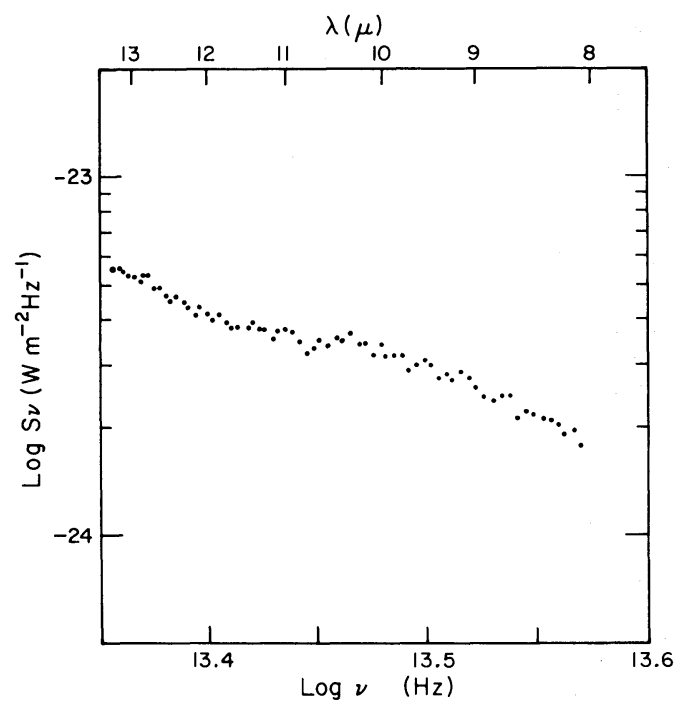

FIG. 3.-Spectrum of the source at 8-13 $\mu$. The data shown are averaged from two scans, with a total of $40 \mathrm{~s}$ integration per point. Statistical errors are $\pm 5 \%$ as determined relative to $\alpha$ Tau, although the absolute flux is less certain. facts that the spectra of the two nebulous objects are similar and that the direction of the polarization vector is compatible with illumination by a central obscured object at the position of the infrared source. The geometry is therefore very similar to that proposed by Ney et al. (1975) for CRL 2688, an infrared source associated with a nebula in Cygnus. This object differs from CRL 2688, however, in showing strong emission lines characteristic of an ionized gas. Models with similar geometries have also been suggested for some of the Herbig-Haro objects by Strom et al. (1974) and for M1-92 by Herbig (1975).

The measured $\mathrm{H} \beta$ flux density can be used to derive an upper limit to the angular size of the ionized region. It is shown below ( $\S \mathrm{III} b$ ) that the emission-line fluxes as measured from the Earth are reddened by about $A_{v}=3.5 \mathrm{mag}$. Furthermore, because of geometric, solid angle, and albedo effects in the reflection nebulosity, not more than one-third of the $\mathrm{H} \beta$ photons produced in the ionized region will be reflected toward the Earth. The measured $\mathrm{H} \beta$ flux density, corrected for these factors corresponds to a free-free flux density at $5 \mathrm{GHz}$ of at least $30 \times 10^{-29} \mathrm{~W} \mathrm{~m}^{-2} \mathrm{~Hz}^{-1}$, which is greater than the observed upper limit at this wavelength. It can therefore be deduced that the $\mathrm{H}$ II region is self-absorbed at $5 \mathrm{GHz}$, that its angular diameter is less than 0.25 , and that its emission measure is in excess of $10^{8} \mathrm{pc} \mathrm{cm}^{-6}$ (Mezger and Henderson 1967). These calculations were made using the data of Pengelly (1964) and an assumed electron temperature of $20,000 \mathrm{~K}(\S \mathrm{III} c)$.

Figure 5 shows a possible model for the geometry of the object. The central star is surrounded by a 0 ". 25 diameter ionized region and a region of heated dust producing the 0".4 diameter infrared source. There is an intervening dust cloud between the star and the Earth, but this does not prevent light from the star and the $\mathrm{H}$ II region being reflected toward the Earth by the 


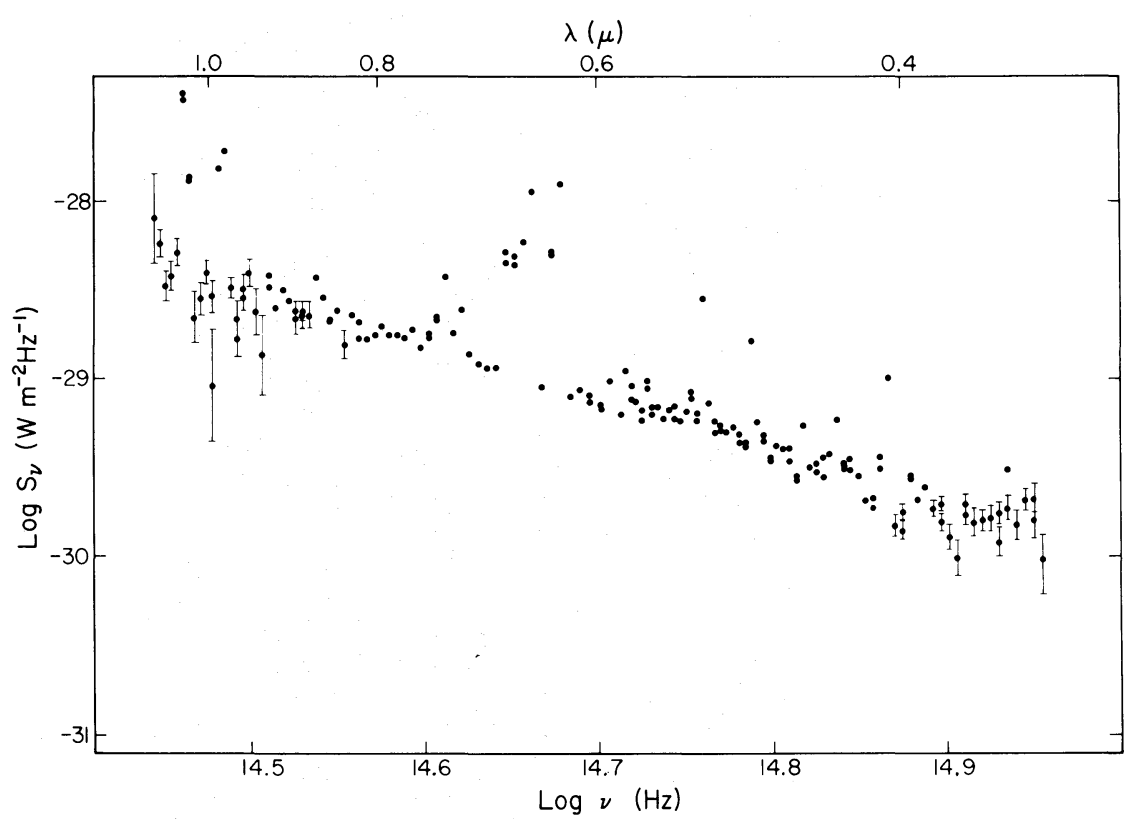

FIG. 4.-Optical spectrum of the object made with the 32-channel spectrophotometer. Points without error bars have errors less than $10 \%$.

two thinner regions of dust to each side. Although drawn as separate regions in Figure 5, the infrared source, the obscuring region, and the reflection nebulae are probably all part of the same dust cloud. The shape of this cloud is uncertain, though the suggestion by Ney et al. (1975) (in the context of CRL 2688), that the obscuring cloud is in the form of a toroid with its axis along a line joining the two reflection nebulosities, is attractive from the point of view of symmetry.

\section{b) Extinction}

The fact that all the visible light is seen by reflection makes it very difficult to estimate the extinction toward CRL 618. Because reflection nebulae are generally bluer than their illuminating stars, we may assume that any extinction derived on the basis of the apparent colors of the object is a lower limit to the correct value.

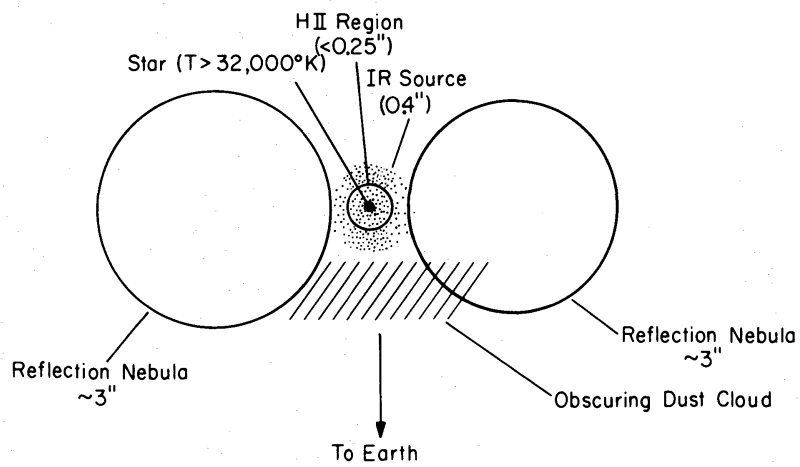

FIG. 5.-The model for the source
For an ionized region which is optically thick in the Lyman lines and has an electron temperature of $20,000 \mathrm{~K}$ (see below), Brocklehurst (1971) predicts hydrogen line intensities relative to $\mathrm{H} \beta$ of $2.74(\mathrm{H} \alpha)$, $0.476(\mathrm{H} \gamma)$, and $0.051(\mathrm{P} \delta)$. Comparison of these values with the measured fluxes (Table 3) and a Whitford extinction law (Johnson 1968) gives a visual absorption of $A_{v}=3.4,4.4$, and $2.6 \mathrm{mag}$, respectively. $A_{v}$ can also be obtained from the ratio of the auroral $(\lambda \lambda 10287$, $10320,10338,10373)$ and the transauroral $(\lambda \lambda 4069$, 4076) lines of [S II] which originate from the same upper level. According to Miller (1968) their ratio should be 0.55 , whereas the value measured for the source is 19 , corresponding to an absorption $A_{v}$ of $3.7 \mathrm{mag}$. This value of $A_{v}$ would be an overestimate if part of the intensity attributed to the infrared lines is actually from [N I] $\lambda 10400$ (see below). We have adopted a mean value of $A_{v}=3.5$ mag for the absorption to the emission-line region.

The optical continuum data may also be used to estimate extinction. The continuum is too strong compared to $\mathrm{H} \beta$ to have been produced by transitions in the ionized gas alone, and moreover does not show significant Balmer or Paschen discontinuities. The continuum is therefore presumably dominated by reflected starlight. The star must be a B type or earlier in order to produce an $\mathrm{H}$ II region, so its intrinsic energy distribution over the wavelength range in Figure 4 is close to a Rayleigh-Jeans law. The extinction to the continuum source calculated by this method on the assumption of a Whitford law is $4.7 \pm 0.4 \mathrm{mag}$, in excess of the extinction to the emission-line region. This result is interpreted as indicating the presence of some dust within the ionizing region. The amount of dust within the ionized region is equivalent to about 
$1 \mathrm{mag}$ in $A_{v}$, and the dust competes with the gas for the absorption of stellar ultraviolet photons (see $\S$ III $d$ ). It is not possible to tell whether the remaining extinction arises in the general interstellar medium or in the vicinity of the source.

It is important to realize that none of the data presented here require extremely large amounts of dust. In fact, the absence of a silicate absorption feature is compatible with a visual extinction $A_{v} \sim 5$ mag in the line of sight to the infrared source (Rieke 1974).

\section{c) Physical Conditions in the Ionized Region}

The electron temperature $T_{e}$ and electron density $N_{e}$ have been derived from optical emission-line intensity ratios. We use predicted ratios as a function of $T_{e}$ and $N_{e}$ for $I(6548+6583) / I(5755)$ of $[\mathrm{N} \mathrm{II}]$ (Osterbrock $1974), \quad I(6716+6730) / I(10287+10320+10338+$ 10373) of [S II] (Czyzak et al. 1970), and I(3726 + 3729)/ $I(7320+7330)$ of [O II] (Peimbert and Costero 1969). The observed intensity ratios of the [N II] and of the [S II] lines, corrected for $A_{v}=3.5 \mathrm{mag}$, yield $T_{e}=$ $20,000 \mathrm{~K}$ and $N_{e}=6 \times 10^{4} \mathrm{~cm}^{-3}$. We would then expect $I(3726+3729) / I(7320+7330)$ of $[\mathrm{O}$ II] to be 1.0, which, after correction for interstellar extinction, is just compatible with the estimated upper limit of the line strength of $0.005 \times 10^{-15} \mathrm{~W} \mathrm{~m}^{-2}$.

The presence of strongly forbidden $\lambda 5199$ emission of $\left[\mathrm{N}_{\mathrm{I}}\right]$ requires that it originate in a region of lower density than that derived above. Using collision strengths derived by Henry and Williams (1968), we find a predicted ratio $I(10400) / I(5199)$ of the order of $2 \times 10^{-4} N_{e}$. The [N I] $\lambda 10400$ emission cannot be resolved from the $[\mathrm{S}$ II] emission at somewhat shorter wavelengths. Perhaps up to one-third of the emission, which in Table 3 has been assigned to [S II], could be contributed by $\left[\mathrm{N}_{\mathrm{I}}\right]$. In that case $I(10400) / I(5199)$, corrected for interstellar extinction, is at most 0.4 and hence $N_{e}<2 \times 10^{3} \mathrm{~cm}^{-3}$. In summary, then, there is evidence for the presence of regions with a large range of electron density, at least from $10^{3}$ to $10^{5} \mathrm{~cm}^{-3}$.

\section{d) The Luminosity and Temperature of the Exciting Star}

Little is known about the distance to the object. Since the radial velocity of the source with respect to the local standard of rest is $-50 \mathrm{~km} \mathrm{~s}^{-1}$, it is extremely probable that it lies within the Galaxy. Its comparatively high galactic latitude and its location toward the anticenter $\left(l^{\mathrm{II}}=166^{\circ} .5, b^{\mathrm{II}}=-6.5\right)$ make it unlikely that the object is more than $5 \mathrm{kpc}$ from the Sun.

If the dust cloud surrounding the star is assumed to be optically thick at visible wavelengths, then the measured infrared bolometric luminosity may be equated to the bolometric luminosity of the star itself. Integration of the energy distribution in Figure 2 leads to the conclusion that the luminosity of CRL 618 shortward of $40 \mu$ is $5 \times 10^{3} R^{2} L_{\odot}$, where $R$ is the distance in kpc. Allowance for radiation longward of
$40 \mu$, assuming a blackbody temperature of $275 \mathrm{~K}$, raises this to $6 \times 10^{3} R^{2} L_{\odot}$.

An approximate estimate may be made of the temperature of the exciting star by comparing its apparent magnitude with the $\mathrm{H} \beta$ flux from the compact $\mathrm{H}$ II region surrounding it. Since, ignoring for the time being the presence of dust within the $\mathrm{H}$ II region, the $\mathrm{H} \beta$ flux in an ionization-bounded nebula is proportional to the number of ionizing photons produced by the central star, the quantity

$$
Y=\log F(\mathrm{H} \beta)+0.4 m_{v}
$$

is a crude ultraviolet to visual color index which is a function mainly of the surface temperature of the exciting star. The quantity $Y$ is obviously independent of the distance to the source; and, since the wavelength of $\mathrm{H} \beta$ is close to that of the $V$-band, it is independent of the fact that the star and nebula are seen only by reflection, and nearly independent of reddening exterior to the ionized region. The quantity $Y$ is, however, dependent on the amount of dust within the ionized region, as discussed below.

The quantity $F(\mathrm{H} \beta)$ is given in Table 3 ; and $m_{v}$, as calculated from the continuum flux density at $5510 \AA$, is 17.0, giving $Y=-9.4$. The quantity $Y$ can be calculated as a function of stellar temperature using the data tabulated by Panagia (1973) for early-type stars. These data indicate a temperature of about $32,000 \mathrm{~K}$ for the star, corresponding to a mainsequence spectral type B0 -09.5. For comparison a B3 star has $Y=-12.33$ and an 04 star $Y=-8.09$.

The presence of dust within the ionized region, which has been demonstrated for this source in $\S$ III $b$, complicates the calculation considerably. Since the $\mathrm{H} \beta$ flux is proportional to the rate of ionization, it is attenuated by an amount corresponding to the absorption by dust of Lyman continuum radiation. So long as the dust particles are smaller than about $0.5 \mu$, therefore, the presence of dust within the ionized region attenuates the $\mathrm{H} \beta$ emission more than the stellar visible emission. The estimate of the stellar temperature $(32,000 \mathrm{~K})$ obtained above is thus a lower limit to the true stellar temperature, although there are too many uncertainties to allow a quantitative correction to be made. The absence of strong helium lines in the spectrum of the $\mathrm{H}$ II region is interesting in this context; either the stellar temperature is too low to ionize helium or else the dust is selectively absorbing the shortest wavelength ultraviolet photons (Mezger et al. 1974).

\section{e) The Nature of the Object}

The fact that the central star of the object is so intimately associated with a dust cloud indicates that it is probably at either a very early or a very late stage in its evolution. The former seems unlikely for two reasons. First, the object appears isolated from other clouds of gas or new stars; second, since a pre-mainsequence object should lie on or above the main sequence, the intrinsic luminosity of this object (given $T^{*} \geq 32,000 \mathrm{~K}$ ) would be greater than $3 \times 10^{4} L_{\odot}$ 
and its distance from the Sun greater than $2.5 \mathrm{kpc}$. This would put the object at least $280 \mathrm{pc}$ from the plane, which is large for what should be an extreme Population I object.

We prefer the hypothesis that CRL 618 is some kind of planetary nebula at a smaller (but unspecified) distance from the Sun. In this way we can explain the high temperature of the star, the presence of a somewhat symmetrical cloud of gas and dust, the emission lines, and the isolation of the object. CRL 618, if a planetary nebula, is unique among such objects in being severely dust-bounded. Absorption of stellar ultraviolet radiation has been detected in other planetaries, such as NGC 7027 (Becklin et al. 1973), but in that case the optical depth of the dust grains was an order of magnitude less than here. The high electron density, compact nature, and especially the large dust optical depth all suggest that CRL 618 is an extremely young planetary nebula, perhaps still in the process of formation. Without a distance to the object we hesitate to quantify this proposal, but suggest instead that searches be made for molecular emission from the neutral gas which may surround the source.

We thank the staffs of the Hale and Mauna Kea Observatories and our many colleagues who helped with the observations and data reduction, particularly Judy Bennett, and also Ed Sutton for building the $34-\mu$ system. We acknowledge valuable discussions as to the nature of the object with Michael Werner, and we thank Guido Münch for advice and for directing our attention to the [Fe II] lines observed in the spectrum of $\eta$ Carinae. This work was supported in part by National Aeronautics and Space Administration grants NGL 05-002-207 and NGL 05-002-134 and National Science Foundation grant GP-35545 X.

\section{REFERENCES}

Becklin, E. E., Neugebauer, G., and Wynn-Williams, C. G. 1973, Ap. J. (Letters), 182, L7.

Brocklehurst, M. 1971, M.N.R.A.S., 153, 471.

Czyzak, S. J., Krueger, T. K., and Aller, L. H. 1970, Proc. Nat. Acad. Sci., 66, 282.

Gillett, F. C., and Forrest, W. J. 1973, Ap. J., 179, 483.

Henry, R. J. W., and Williams, R. E. 1968, Pub. A.S.P., 80, 669.

Herbig, G. 1975, Ap. J., $200,1$.

Johnson, H. L. 1968, in Nebulae and Interstellar Matter, ed. B. M. Middlehurst and L. H. Aller (Chicago: University of Chicago Press), p. 167.

Mezger, P. G., and Henderson, A. P. 1967, Ap. J., 147, 471.

Mezger, P. G., Smith, L. F., and Churchwell, E. 1974, Astr. and Ap., 32, 269.

Miller, J. S. 1968, Ap. J. (Letters), 154, L57.

Ney, E. P., Merrill, K. M., Becklin, E. E., Neugebauer, G., and Wynn-Williams, C. G. 1975, Ap. J. (Letters), 198, L129.

Osterbrock, D. E. 1974, Astrophysics of Gaseous Nebulae (San Francisco: Freeman).

Panagia, N. 1973, A.J., 78, 929.

Peimbert, M., and Costero, R. 1969, Bol. Obs. Tonantzintla y Tacubaya, 5, 3.

Pengelly, R. M. 1964, M.N.R.A.S., 127, 145.

Rieke, G. H. 1974, Ap. J. (Letters), 193, L81.

Ryle, M. 1972, Nature, 239, 435.

Strom, S. E., Grasdalen, G. L., and Strom, K. M. 1974, Ap. J., 191, 111.

Thackeray, A. D. 1953, M.N.R.A.S., 113, 211.

E. E. Becklin, G. Neugebauter, and S. P. Willner: George W. Downs Laboratory of Physics (320-47), California Institute of Technology, Pasadena, CA 91125

K. M. MerriLl: Department of Physics, University of California, San Diego, La Jolla, CA 92037

M. SснмidT: Robinson Laboratory (105-24), California Institute of Technology, Pasadena, CA 91125

C. G. Wynn-Williams: Mullard Radio Astronomy Observatory, Cavendish Laboratory, Madingley Road, Cambridge CB3 OHE, England 